\title{
Fecal S100A12 levels measured by a new ELISA are increased in ulcerative colitis (UC) and Crohn's disease $(C D)$ and correlates with intestinal damage
}

\author{
P Garnero ${ }^{1 *}$, C Préaudat $^{1}$, S Vermeire ${ }^{2}$ \\ From 5th European Workshop on Immune-Mediated Inflammatory Diseases \\ Sitges-Barcelona, Spain. 1-3 December 2010
}

\section{Introduction}

Inflammatory bowel diseases (IBDs) are characterized by inflammation and intestinal damage. The $\mathrm{S} 100$ proteins S100A8/A9 and S100A12 have been suggested to be useful for assessing disease activity and monitor treatment efficacy. S100A8/A9 is produced by granulocytes, monocytes and macrophages whereas S100A12 expression is restricted to granulocytes and may thus be more specific.

\section{Aim}

To develop an ELISA for fecal S100A12 and investigate its utility for the clinical investigation of patients with $\mathrm{UC}$ and $\mathrm{CD}$.

\section{Patients and methods}

The ELISA (Inflamark ${ }^{\mathrm{ma}}$, Cisbio bioassays) is based on 2 monoclonal antibodies raised against recombinant human S100A12. After stool extraction, S100A12 was measured in UC $(n=30), C D(n=30)$ and healthy $(n=30)$ subjects. Fecal S100A12 was correlated with the endoscopic Mayo score in subjects with UC and data were compared to fecal S100A8/A9.

\section{Results}

Western blotting showed that the two antibodies selected for the ELISA recognized both recombinant and purified native human S100A12 and did not crossreact with S100A8/A9. Intra and inter assay precision errors were below 10\%. Fecal S100A12 levels were significantly higher in UC (median: $167 \mu \mathrm{g} / \mathrm{g}$ stool, $\mathrm{p}<0.0001$ ) and $\mathrm{CD}$ (median $14 \mu \mathrm{g} / \mathrm{g}$ stool, $\mathrm{p}<0.0001$ ) patients than in healthy controls (median: 0 ), with a corresponding area under the ROC of $0.83(\mathrm{p}<0.0001)$ and 0.71 ( $\mathrm{p}<.0001$ ), in UC and CD, respectively. In patients with UC, fecal S100A12 correlated with the endoscopic Mayo score $(\mathrm{r}=0.48, \mathrm{p}=0.0067)$ and levels were on average 24 fold higher in subjects presenting with active $(\mathrm{n}=16$, Mayo score 2-3) than in patients with inactive $(n=14$, Mayo score 0-1) disease. The difference between active and non-active UC patients was lower (5.2 fold) for S100A8/9 than for S100A12 and did not reach statistical significance $(\mathrm{p}=0.067)$.

\section{Conclusions}

The new ELISA for fecal S100A12 is precise and could detect increased inflammatory activity in patients with UC and CD. Measurements of fecal S100A12 should be useful for the non-invasive assessment of intestinal damage in patients with IBDs.

\section{Author details \\ ${ }^{1}$ Cisbio Bioassays, Bagnols/Cèze, France. ${ }^{2}$ University Hospital Gasthuisberg, Leuven, Belgium.}

Published: 25 November 2010

doi:10.1186/1479-5876-8-S1-P40

Cite this article as: Garnero et al:: Fecal S100A12 levels measured by a new ELISA are increased in ulcerative colitis (UC) and Crohn's disease (CD) and correlates with intestinal damage. Journal of Translational Medicine 2010 8(Suppl 1):P40. 